\title{
Memory-enhancing intra-basolateral amygdala infusions of clenbuterol increase Arc and CaMKIl $\alpha$ protein expression in the rostral anterior cingulate cortex
}

\section{Crystal M. Holloway-Erickson ${ }^{1}$, Jayme R. McReynolds ${ }^{2}$ and Christa K. McIntyre ${ }^{2 *}$}

${ }^{1}$ Department of Psychology, The University of Michigan, Ann Arbor, MI, USA

2 School of Behavioral and Brain Science, The University of Texas at Dallas, Richardson, TX, USA

Edited by:

Antonella Gasbarri, University of L'Aquila, Italy

\section{Reviewed by:}

Clive R. Bramham, University of Bergen, Norway

Maria-Isabel Miranda, Universidad

Nacional Autónoma de México,

Mexico

${ }^{*}$ Correspondence:

Christa K. McIntyre, School of

Behavioral and Brain Sciences, The

University of Texas at Dallas, $800 \mathrm{~W}$

Campbell Road, Richardson, TX

75080, USA.

e-mail:Christa.mcintyre@

utdallas.edu

Activation of $\beta$-adrenoceptors in the basolateral complex of the amygdala (BLA) modulates memory through interactions with multiple memory systems. The cellular mechanisms for this interaction remain unresolved. Memory-modulating BLA manipulations influence expression of the protein product of the immediate early gene activity-regulated cytoskeletal-associated protein (Arc) in the dorsal hippocampus, and hippocampal expression of Arc protein is critically involved in memory consolidation and long-term potentiation. The present studies examined whether this influence of the BLA is specific to the hippocampus and to Arc protein. Like the hippocampus, the rostral portion of the anterior cingulate cortex ( $\mathrm{ACC}$ ) is involved in the consolidation of inhibitory avoidance (IA) memory, and IA training increases Arc protein in the $\mathrm{rACC}$. Because the BLA interacts with the rACC in the consolidation of IA memory, the rACC is a potential candidate for further studies of BLA modulation of synaptic plasticity. The alpha isoform of the Calcium/Calmodulin-dependent protein kinase II (CaMKII $\alpha$ ) and the immediate early gene c-Fos are involved in long-term potentiation and memory. Both Arc and CaMKIl $\alpha$ proteins can be translated in isolated synapses, where the mRNA is localized, but c-Fos protein remains in the soma. To examine the influence of memory-modulating manipulations of the BLA on expression of these memory and plasticity-associated proteins in the rACC, male Sprague-Dawley rats were trained on an IA task and given intra-BLA infusions of either clenbuterol or lidocaine immediately after training. Findings suggest that noradrenergic stimulation of the BLA may modulate memory consolidation through effects on both synaptic proteins Arc and CaMKIl $\alpha$, but not the somatic protein c-Fos. Furthermore, protein changes observed in the rACC following BLA manipulations suggest that the influence of the BLA on synaptic proteins is not limited to those in the dorsal hippocampus.

Keywords: synaptic plasticity, stress, emotion, memory consolidation, immediate early gene, local translation, norepinephrine, noradrenalin

\section{INTRODUCTION}

Stressful or emotionally arousing events are typically remembered better than emotionally neutral events. Stress hormones, released by the adrenal glands into the bloodstream, assist in preparing an animal to fight or flee by increasing energy resources and promoting attention and vigilance. Extensive evidence indicates that this sympathetic response contributes to the enhancement of memory consolidation through actions on $\beta$-adrenoceptors in the basolateral complex of the amygdala (BLA) (Liang et al., 1986; Quirarte et al., 1997; McIntyre et al., 2002; McReynolds et al., 2010). A single footshock is sufficient to produce a longterm contextual memory in rats and increases norepinephrine (NE) levels in the amygdala (Quirarte et al., 1998; McIntyre et al., 2002). Administration of an antagonist to $\beta$-adrenoceptors in the BLA blocks the memory enhancement produced by systemic administration of epinephrine (Liang et al., 1986) or the glucocorticoid corticosterone (Quirarte et al., 1997; McReynolds et al., 2010). Further, direct activation of the noradrenergic system in the amygdala, through infusions of NE or the $\beta$-adrenoceptor agonist clenbuterol enhance memory of inhibitory avoidance (IA) training, passive avoidance tasks, contextual fear conditioning, conditioned taste aversion, and object recognition training (Gold and van Buskirk, 1975; Gallagher et al., 1977; Ferry and McGaugh, 1999; Hatfield and McGaugh, 1999; LaLumiere et al., 2003; Miranda et al., 2003; McIntyre et al., 2005; Roozendaal et al., 2008).

The amygdala appears to interact with many other brain areas in order to influence these various types of memories (McGaugh, 2004). In fact, memory processing involving the hippocampus (Packard et al., 1994; Malin and McGaugh, 2006), caudate nucleus (Packard et al., 1994), insular cortex (Miranda and McGaugh, 2004), entorhinal cortex (Roesler et al., 2002), medial prefrontal cortex (mPFC) (Roozendaal et al., 2009) and the anterior cingulate cortex (Malin and McGaugh, 2006; Malin et al., 2007) is modulated by manipulations of the amygdala. These findings suggest that the BLA may naturally exercise control over synaptic 
plasticity in other regions of the brain that are engaged in memory consolidation.

Expression of the activity-regulated cytoskeletal-associated protein $(A r c)$ immediate early gene can be used as a marker for neuronal plasticity based on its rapid appearance and degradation, and the critical involvement of Arc protein in long-term synaptic plasticity and memory (Bramham et al., 2008). Pre-training intra-hippocampal infusions of antisense oligodeoxynucleotides (ODNs), which disrupt translation of Arc protein, impair longterm memory of spatial water maze, and IA training (Guzowski et al., 2000; McIntyre et al., 2005). Infusions of the $\beta$-adrenoceptor agonist clenbuterol into the BLA immediately after training on a single-trial IA task enhance both memory retention and expression of Arc protein in the dorsal hippocampus (McIntyre et al., 2005). Intra-BLA infusions of the sodium channel blocker lidocaine into the BLA immediately after training impair memory for IA and decrease Arc protein expression in the dorsal hippocampus. These findings support the hypothesis that the BLA modulates the consolidation of long-term memory through actions on efferent brain regions such as the hippocampus.

Arc is also expressed in areas of the brain outside of the hippocampus. Both aversive and non-aversive tasks increase Arc mRNA in cortical and limbic areas of the brain (Kelly and Deadwyler, 2002, 2003; Ons et al., 2004; Ploski et al., 2008). In mice, fear conditioning increases Arc mRNA in the hippocampus as well as the anterior cingulate cortex, the olfactory bulb, the pyriform, parietal, sensory and motor cortices, and the amygdala (Montag-Sallaz and Montag, 2003). Similarly, stress and fear conditioning increase Arc protein expression in many of the same areas of the rat brain, as well as the mPFC (Kelly and Deadwyler, 2003; Koya et al., 2005; Mikkelsen and Larsen, 2006; Ploski et al., 2008). However, it is unknown whether the BLA can influence these training-induced changes in Arc expression in areas outside of the hippocampus.

The immediate early gene Arc has received attention due to the presence of mRNA in dendritic spines, where it can be translated to protein by interacting with local ribosomes (Steward and Worley, 2002; Yin et al., 2002; Moga et al., 2004; Bramham et al., 2008). Based on evidence that Arc protein is involved in synaptic plasticity, this expression pattern presents a possible mechanism for synapse-specific modifications. However, Arc is not the only known memory-related immediate early gene, or locally translated protein. Our previous findings suggest that the BLA does not modulate expression of the protein product of the immediate early gene c-Fos in the dorsal hippocampus (McIntyre et al., 2005). Both c-Fos protein and mRNA are localized to the soma. Therefore, it is possible that the BLA influences memory through synapse-specific effects by modulating local translation of synaptically localized mRNAs. Another locally translated protein is the alpha-isoform of Calcium/Calmodulin-dependent protein kinase II $(\mathrm{CaMKII} \alpha)$, which appears to play a similar role in memory (Silva et al., 1992a,b). If the BLA modulates memory through an influence specifically on local translation of synaptic proteins, then memory-modulating stimulation of the BLA should produce changes in Arc and CaMKII $\alpha$, but not c-Fos protein.

Like the hippocampus, the rostral portion of the anterior cingulate cortex (rACC) appears to interact with the amygdala to consolidate memory of IA training (Malin and McGaugh, 2006; Malin et al., 2007). Activating the rACC with the muscarinic agonist oxotremorine immediately after IA training enhances memory for the nociceptive component of the task, as evidenced by longer retention latencies when tested $48 \mathrm{~h}$ later (Malin and McGaugh, 2006). When the BLA is lesioned prior to activation of the rACC by oxotremorine, the memory-enhancing effect is attenuated, showing an interaction between the rACC and the BLA for long-term consolidation of memory (Malin et al., 2007). Indeed, there is substantial evidence showing direct anatomical connections between the ACC and the BLA (Sarter and Markowitsch, 1983; Sripanidkulchai et al., 1984; McDonald, 1991). We recently reported that Arc protein in synapses of the rACC is increased following IA training and rACC expression of Arc protein is necessary for optimal long-term memory for the task (Holloway and McIntyre, 2011). Using western immunoblotting, we found a significant increase in Arc protein expression in tissue taken from the rACC of rats that were trained on a single-trial IA task, compared to the rACC of naive rats. Infusions of antisense ODNs into the rACC immediately or $6 \mathrm{~h}$ after IA training disrupted memory for the task (Holloway and McIntyre, 2011). These results provide a basis for exploring the effect of post-training stimulation of BLA $\beta$-adrenoceptors on expression of long-term memory and synaptic plasticity-associated proteins Arc, CamKII $\alpha$, and c-Fos in the rACC.

\section{METHODS}

\section{SUBJECTS}

One hundred and fifty four male Sprague-Dawley rats (250-275 g upon arrival) were purchased from Charles River Laboratories (Wilmington, MA). All animals were housed separately in a temperature controlled setting $\left(19.5^{\circ} \mathrm{C}\right)$ maintained on a $12 \mathrm{~h}$ light/dark cycle (lights on at 7:00 AM), given food and water ad libitum. All procedures were conducted in accordance with National Institutes of Health guidelines and approved by The University of Texas at Dallas Institutional Animal Care and Use Committee.

\section{SURGERIES}

Rats were anesthetized with isoflurane $\left(1 \%\right.$ in $\mathrm{O}_{2}$, Western Medical Supply). A continuous flow of isoflurane was delivered to the animal throughout the surgery. Once anesthetized, rats' heads were leveled in a stereotaxic device (Stoelting Inc, Wood Dale, IL). Rats were given subcutaneous injections of $0.5 \mathrm{~mL}$ marcaine/lidocaine at the incision site. Fifteen millimeter cannulae were placed directly above the BLA [Coordinates in $\mathrm{mm}$ : anteroposterior (AP), -2.7 from bregma; mediolateral $(\mathrm{ML}) \pm$ 5.2 from the midline; dorsoventral (DV) -6.4 from the top of the skull; incisor bar, $-3.3 \mathrm{~mm}$ from interaural line (Paxinos and Watson, 2005)], mounted with dental cement and secured with skull screws. Stylets ( $15 \mathrm{~mm}$ long insect dissection pins) were inserted into each cannula to maintain patency. Animals were given $3-5 \mathrm{~mL}$ of saline subcutaneously to help prevent dehydration, and moved to a warm recovery chamber until they were awake and moving. They were then allowed to recover for one week before training. 


\section{INHIBITORY AVOIDANCE (IA) TRAINING}

All rats were handled for $2 \mathrm{~min} /$ day for five days prior to being trained on an IA task. The IA apparatus was a trough-shaped box, $90 \mathrm{~cm}$ in length, with a sliding guillotine style door dividing two compartments. The box was placed in a dark room, and the rats were brought into the room immediately before training. The lighted compartment was $30 \mathrm{~cm}$ long, with white plastic sides. A table lamp sat directly over this side, shining down into the compartment. The dark compartment was $60 \mathrm{~cm}$ long, with metal sides. Each rat was removed from its home cage and placed in the light compartment facing away from the dark compartment. Once the rat turned around $\left(180^{\circ}\right)$ and crossed over into the dark compartment, the sliding guillotine door was closed and the rat was trapped in the dark compartment. When the rat walked all the way to the end of the box and turned around again, an inescapable $1.0 \mathrm{~s}$ shock $(0.38 \mathrm{~mA}$ for clenbuterol-infused rats; $0.48 \mathrm{~mA}$ for lidocaine-infused rats) was applied to the floor plates. The rat remained in the dark compartment for $10 \mathrm{~s}$ following the shock before being removed from the IA box and given immediate, post-training intra-BLA infusions of either clenbuterol or lidocaine. Rats were returned to the IA apparatus for memory retention testing $48 \mathrm{~h}$ after completion of training. They were again placed in the light compartment. Latency to cross into the dark compartment was recorded and used as a measure of memory. If a rat failed to cross into the dark compartment before ten minutes, it was removed and a time of $600 \mathrm{~s}$ was recorded.

\section{INFUSIONS}

The $\beta$-adrenoceptor agonist clenbuterol $(4 \mathrm{ng} / 0.2 \mu \mathrm{L}$ dissolved in a vehicle of $0.9 \%$ saline; Sigma-Aldrich), the local anesthetic lidocaine $(2.0 \%$ in saline; Hospira Inc., Lake Forest, IL), or vehicle were administered immediately post-training, through 30 gauge dental needles-extending $2 \mathrm{~mm}$ beyond the cannulae-attached to $10 \mu \mathrm{L}$ Hamilton microsyringes. A volume of $0.2 \mu \mathrm{L}$ of drug or vehicle was administered over $32 \mathrm{~s}$, at a constant rate, by a KD Scientific (Harvard Instruments) infusion pump. Infusion needles remained in place for an additional $30 \mathrm{~s}$ to allow for diffusion of the drug. The doses of clenbuterol and lidocaine were used based on results of previous research showing their effectiveness at enhancing or impairing memory for an IA task (ColemanMesches and McGaugh, 1995; McIntyre et al., 2005). For the behavioral task, animals received bilateral post-training intraBLA infusions. For analysis of protein expression in the ACC, rats were given infusions of clenbuterol or lidocaine into the right or left BLA (and vehicle into the other hemisphere), or vehicle bilaterally (Figures 3-5). In this way, each rat served as its own control (as reported previously in McIntyre et al., 2005).

\section{TISSUE PREPARATION}

Animals were deeply anesthetized using isoflurane (Western Medical Supply) and brains were rapidly removed and flash frozen by submersion in 2-methylbutane in a dry ice/ethanol bath. Trained animals were euthanized $1 \mathrm{~h}$ after training. This time point was chosen based on previous research showing increases in Arc, c-Fos, and CaMKII $\alpha$ protein in the hippocampus or cortex at this time point (Kleim et al., 1996; Ouyang et al., 1999; Otmakhov et al., 2004; Aslam et al., 2009; Holloway and
McIntyre, 2011). The brains were cut coronally just in front of the BLA $(-1.20 \mathrm{~mm}$ from Bregma) and the anterior portions were saved for tissue collection from the ACC. Only brains with correct cannula placement were used. To determine cannula placement, $40 \mu \mathrm{m}$ sections were taken from the posterior section with a cryostat, mounted on gelatin subbed slides, and stained with thionin. These slides were analyzed under a light microscope to determine the location of the cannulae and drug infusion sites; Figure 1 shows a representative photomicrograph indicating a cannula track and drug infusion site in the BLA. Any brains that did not have needle tips in the BLA were not used for data analysis. A series of $500 \mu \mathrm{m}$ cryosections were collected starting $+4.2 \mathrm{~mm}$ from bregma and continuing to $+2.1 \mathrm{~mm}$ from bregma. The ACC was dissected out using a tissue punch kit $(0.5 \mathrm{~mm}$ diameter $)$, $0.5 \mathrm{~mm}$ from midline to the medial edge of each hemisphere and
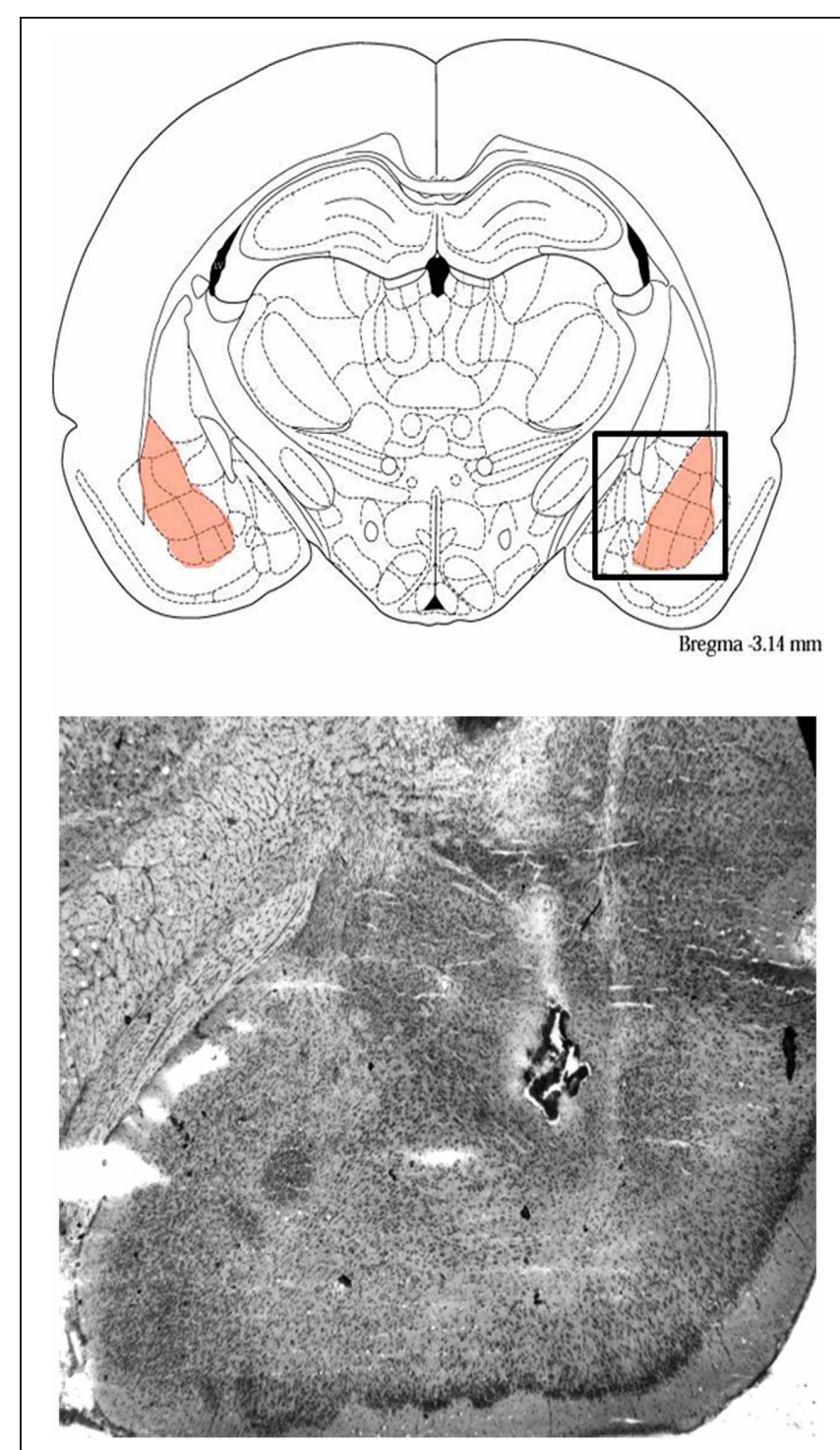

FIGURE 1 | Representative photomicrograph showing cannula tracks and drug infusion site in the BLA, and schematic showing the BLA and surrounding areas. 
starting from the top of the brain and continuing $-2 \mathrm{~mm}$. The tissue punches were stored at $-80^{\circ} \mathrm{C}$ for later western blot analysis.

\section{WESTERN IMMUNOBLOTTING}

Tissue was sonicated in a $0.1 \mathrm{M}$ phosphate buffer, $\mathrm{pH} 7.4$ containing $10 \%$ glycerol, $20 \%$ protease inhibitor cocktail (SigmaAldrich), and $10 \%$ phosphatase inhibitor (Sigma-Aldrich). Protein amounts were determined using a Qubit fluorometer and Qubit protein assay kit (Invitrogen). Approximately $15 \mu \mathrm{g}$ of protein from each sample was heated with a sample buffer and reducing agent (Invitrogen), loaded and then run on $4-12 \%$ BisTris MIDI Gels (Invitrogen) using an electrophoresis apparatus. Each gel contained samples from each experimental condition. Gels were transferred by electroblotting onto a nitrocellulose membrane using an iBlot dry-blotting system (Invitrogen). The membrane was then washed in Tris-buffered saline (TBS: $150 \mathrm{mM}$ $\mathrm{NaCl} / 100 \mathrm{mM}$ tris base, $\mathrm{pH} 7.5$ ) and incubated with primary antibodies diluted in blocking solution (5\% non-fat dry milk in TBS-Tween) overnight at $4^{\circ} \mathrm{C}$. The primary antibodies were AntiArc (rabbit polyclonal; 1:2000, Synaptic Systems), Anti-CaMKII $\alpha$ (rabbit polyclonal; 1:1000, Cell Signaling), Anti-c-Fos (rabbit polyclonal; 1:250, Santa Cruz Biotechnology) and Anti-Actin (rabbit; 1:1500, Sigma-Aldrich). Two days later the membranes were washed in TBS-Tween, and incubated at room temperature for $1 \mathrm{~h}$ in a secondary HRP-linked antibody (goat antirabbit; 1:6000, Cell Signaling). Immunoreactivity was detected using chemiluminescence (ECL Western Blot Kit; Pierce). A marker (Invitrogen) was run to determine the relative mobility of immunoreactive bands. For densitometric quantification, films were scanned and converted into TIF files to be analyzed using Image-J software (NIH).

\section{STATISTICAL ANALYSIS}

Two-sample $t$-tests were used to analyze IA retention latencies in experiment one. Pair-wise comparisons were made between clenbuterol- or lidocaine-treated animals and vehicle-treated rats. For western blot densitometry, results are expressed as a ratio of Arc, CaMKII $\alpha$, and c-Fos to actin, and then expressed as a ratio of drug-infused to vehicle-infused hemisphere, or randomized as a ratio of one hemisphere to the other hemisphere in rats given bilateral vehicle infusions. These values are presented as percent of cage control values to account for film variation. For experiments comparing trained animals to untrained animals, the values are expressed as a percent of experimental/control vehicle group. The final values were analyzed using a Student's $t$-test to make pair-wise comparisons between the groups. A probability level of $p<0.05$ was considered significant. Data are presented as means \pm SEM.

\section{RESULTS}

\section{POST-TRAINING INTRA-BLA INFUSIONS OF CLENBUTEROL OR LIDOCAINE SIGNIFICANTLY ENHANCE OR IMPAIR INHIBITORY AVOIDANCE MEMORY}

In order to investigate the effects of BLA manipulation on memory, rats were trained on the IA task and received immediate bilateral intra-BLA infusions of clenbuterol $(n=7)$ or vehicle $(n=6)$. Memory retention was tested $48 \mathrm{~h}$ later.
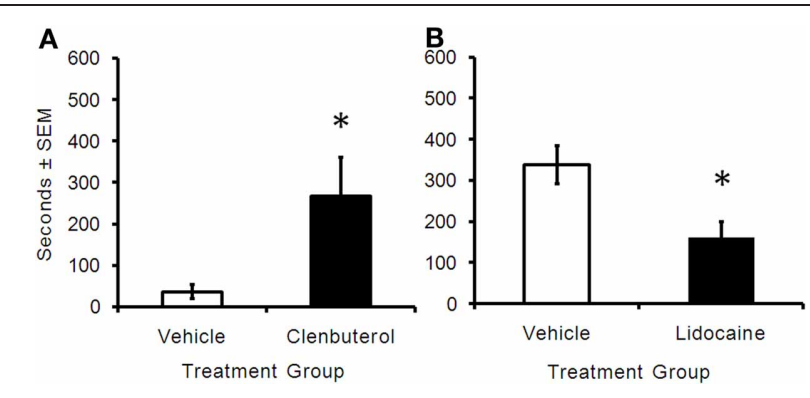

FIGURE 2 | (A) Retention latency times (in seconds) of animals trained on a one-trial inhibitory avoidance task and immediately given intra-BLA infusions of clenbuterol or vehicle. Rats that received infusions of clenbuterol ( $m=267.43 \pm 93.36, n=7$ ) had a significantly greater cross-over latency $48 \mathrm{~h}$ later when compared to animals receiving vehicle infusions ( $m=36 \pm 16.96, n=6)$ ) ${ }^{*} p<0.05$. (B) Rats given post-training intra-BLA infusions of lidocaine ( $m=162 \pm 36.91, n=13$ ) had significantly lower cross-over latencies than animals given vehicle infusions ( $m=338.69 \pm 46.04, n=13) .{ }^{*} p<0.05$.

Clenbuterol-treated rats showed significantly higher retention latencies $($ mean $=267.42 \mathrm{~s})$ than vehicle-treated rats (mean $=$ $36.00 \mathrm{~s}$; Figure $2 \mathrm{~A} ; t(6)=-2.44, p<0.05$ ), suggesting that clenbuterol-infused animals had an enhanced memory for the task $(p<0.05)$. In another experiment, rats were trained on the IA task and received immediate intra-BLA infusions of lidocaine $(n=13)$ or vehicle $(n=13)$. Rats given intra-BLA infusions of lidocaine showed significantly lower retention latencies (mean $=162.00 \mathrm{~s})$ than rats given intra-BLA infusions of vehicle $($ mean $=338.70 \mathrm{~s} ;$ Figure 2B; $t(23)=2.99, p<0.05)$ indicating that lidocaine treatment was memory impairing.

\section{ARC AND CAMKII $\alpha$ PROTEIN LEVELS ARE MODULATED BY INTRA-BLA CLENBUTEROL OR LIDOCAINE INFUSIONS}

To examine the interaction between the BLA and the rACC, separate groups of rats were trained on an IA task and given unilateral infusions of clenbuterol or lidocaine into one hemisphere and vehicle into the other hemisphere, or bilateral intra-BLA vehicle infusions. Arc protein expression in the rACC was measured and reported as a ratio of the drug infused hemisphere/vehicle infused hemisphere. Arc protein expression was significantly increased in rACC tissue taken from the clenbuterol infused rats $(n=10)$ when compared to rats $(n=8)$ given bilateral intra-BLA vehicle infusions (Figure 3A; $t(15)=-3.72, p<0.05$ ). Rats given unilateral infusions of lidocaine $(n=5)$ into the BLA showed decreased Arc protein expression in homogenate tissue collected from the rACC compared to the rats $(n=5)$ given bilateral vehicle infusions (Figure 3B; $t(8)=2.96, p<0.05$ ).

Post-training infusions of the memory-enhancing dose of clenbuterol in the BLA also increased CaMKII $\alpha$ protein expression in the rACC (Figure 4A; $t(9)=-1.94 ; p<0.05$ ). Expression of CaMKII $\alpha$ protein was significantly greater in rats given post-training infusions of clenbuterol $(n=5)$ than vehicletreated controls $(n=6)$. Likewise, CaMKII $\alpha$ protein expression in tissue collected from the rACC was significantly lower in rats given intra-BLA lidocaine infusions $(n=7)$ than vehicle-treated controls $(n=7)($ Figure 4B; $t(12)=3.18 ; p<0.05)$. 


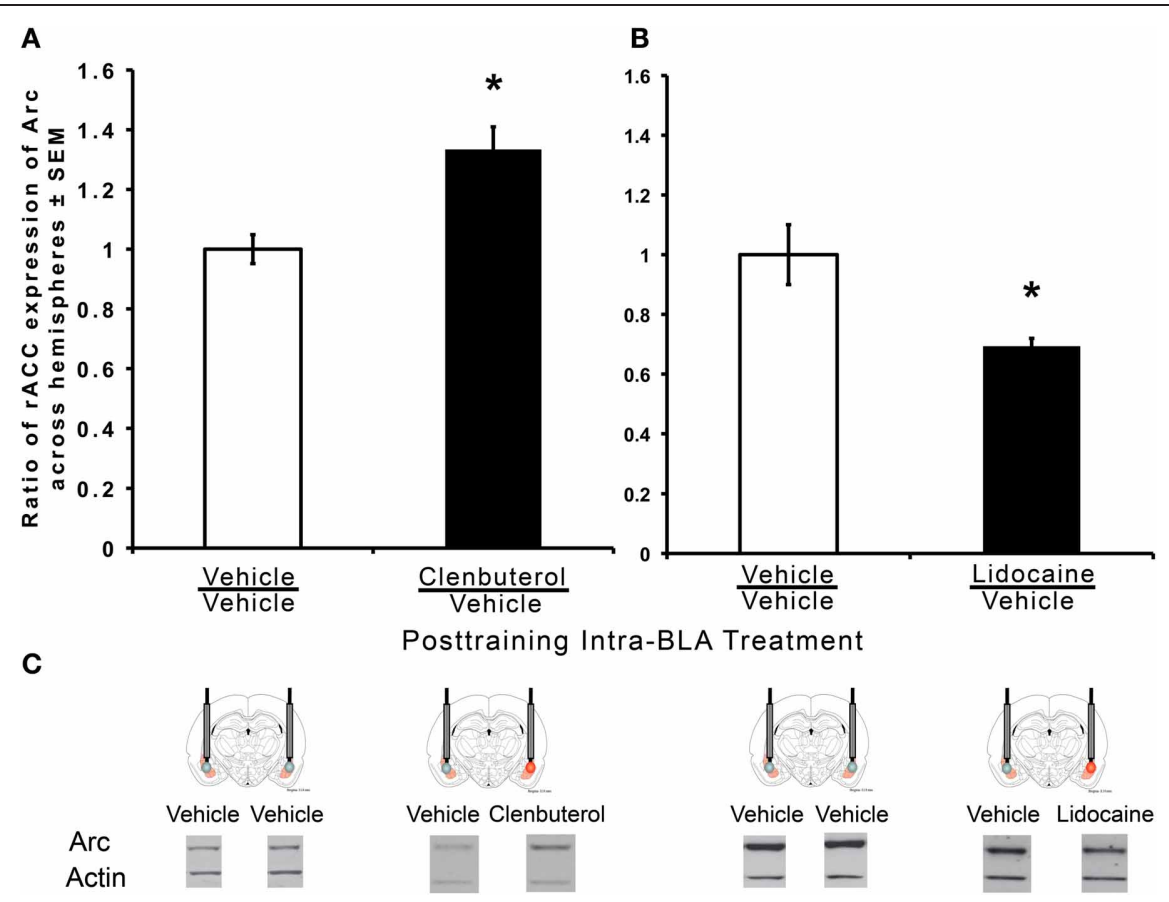

FIGURE 3 | Memory-enhancing intra-BLA infusions of clenbuterol increase Arc expression and memory-impairing intra-BLA infusions of lidocaine decrease Arc expression in the rACC. Western immunoblotting was used to quantify protein expression in the $\mathrm{rACC}$ following inhibitory avoidance training. (A) Significantly greater Arc protein expression was measured in the $\mathrm{rACC}$ of rats treated with intra-BLA infusions of clenbuterol immediately following training ( $m=1.33 \pm 0.07, n=10)$ when compared to vehicle-infused animals ( $m=1.00 \pm 0.05, n=8$ ). Values are expressed as percent to normalized cage control ratios. ${ }^{*} p<0.01$. (B) Significantly less Arc protein expression was measured in the $\mathrm{rACC}$ of rats treated with intra-BLA infusions of lidocaine immediately after training $(m=0.69 \pm 0.03$, $n=5$ ) compared to vehicle-treated animals ( $m=1.00 \pm 0.10, n=5$ ). Values are expressed as percent to normalized cage control ratios. $* p<0.01$.

\section{C-FOS PROTEIN LEVELS ARE NOT INFLUENCED BY INTRA-BLA CLENBUTEROL OR LIDOCAINE INFUSIONS}

In order to determine whether BLA manipulations have an effect on $\mathrm{c}$-Fos protein expression, rats were trained on the IA task and were given immediate post-training intra-BLA infusions of either clenbuterol or lidocaine into one hemisphere and vehicle into the other or bilateral intra-BLA vehicle infusions. IntraBLA infusions of clenbuterol did not significantly influence c-Fos expression (Figure 5A; $t(9)=0.25, p=0.40$ ). Similarly, intraBLA infusions of lidocaine did not influence $c$-Fos protein expression in the rACC (Figure 5B; $t(11)=-0.67, p=0.25)$. These results led us to question the involvement of $\mathrm{c}$-Fos in the rACC in memory for an IA task. Therefore, c-Fos protein levels were compared in tissue from the rACC of rats trained on an IA task $(n=4)$ to animals that were not trained $(n=4)$. Expression of cFos protein was significantly greater in the rACC of rats that were trained on the task as compared to the naïve cage control animals (Figure 5C; $t(4)=-2.39, p<0.05)$, indicating that c-Fos in the rACC is responsive to IA training, but not to manipulations of the BLA.

\section{DISCUSSION}

The main finding of these experiments is that post-training infusions of a memory-enhancing dose of clenbuterol into the BLA increase expression of the plasticity-associated proteins Arc and CaMKII $\alpha$ in the rACC. Conversely, infusions of lidocaine into the
BLA decrease expression of these synaptic proteins in the rACC. This is consistent with previous findings indicating that the BLA modulates Arc protein expression in the dorsal hippocampus. Taken together, these results suggest that amygdala actions may influence cellular processes involved in the storage of memory by a mechanism that is conserved, at least in part, across brain regions. Results also suggest that the effect of BLA actions is not specific to the immediate early gene Arc.

Consistent with previous findings, bilateral post-training infusions of clenbuterol into the BLA-enhanced, and bilateral intraBLA infusions of lidocaine impaired long-term memory for the IA task (Ferry and McGaugh, 1999; Vazdarjanova and McGaugh, 1999; McIntyre et al., 2005). Using a retrograde tracer, Sripanidkulchai and colleagues demonstrated that BLA projections to the ACC are predominantly ipsilateral (1984). To examine protein expression in the current studies, the memorymodulating doses of clenbuterol or lidocaine were infused unilaterally so proteins in the rACC ipsilateral to the drug infusion could be compared to those in the rACC ipsilateral to an intraBLA vehicle infusion. Therefore, each animal served as its own control. This paradigm is useful as individual differences in protein levels may obscure subtle group differences (McIntyre et al., 2005).

The research carried out here was based on the hypothesis that the BLA modulates the expression of proteins important for the strengthening of synapses that enables the rapid consolidation of 


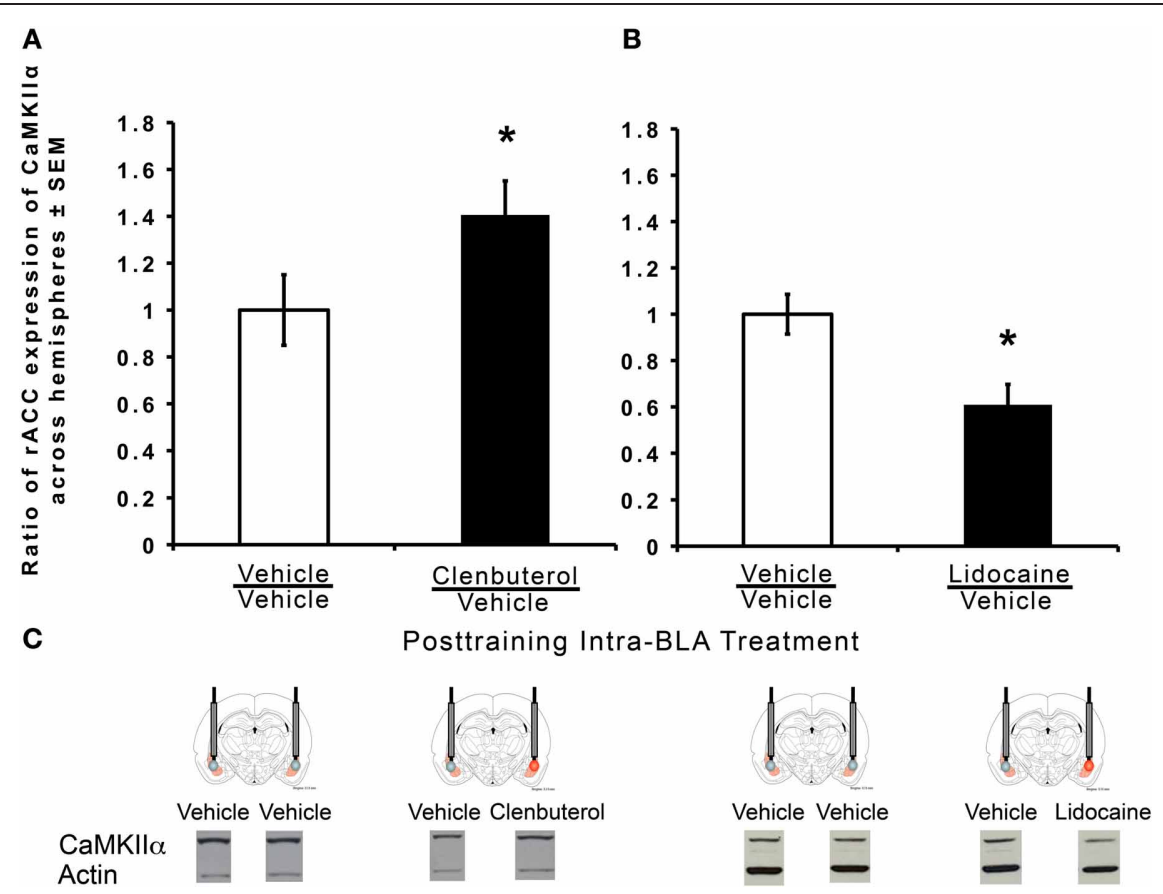

FIGURE 4 | Memory-enhancing intra-BLA infusions of clenbuterol increase CamKIl $\alpha$ expression and memory-impairing intra-BLA infusions of lidocaine decrease CaMKIl $\alpha$ expression in the rACC. Western immunoblotting was used to quantify protein expression in the rACC following inhibitory avoidance training. Values are expressed as percent to normalized cage control ratios. (A) Significantly greater CaMKIl $\alpha$ protein expression was measured in the $\mathrm{rACC}$ of rats treated with infusions of clenbuterol immediately following training ( $m=1.40 \pm 0.14, n=5$ ) when compared to vehicle-infused animals $(1.00 \pm 0.15, n=6) .{ }^{*} p<0.05$. (B) Significantly less CaMKIl $\alpha$ protein expression was measured in the $\mathrm{AACC}$ of rats treated with intra-BLA infusions of lidocaine immediately after training ( $m=0.61 \pm 0.09$, $n=7$ ) compared to vehicle-infused animals ( $m=1.00 \pm 0.09, n=7$ ). $* p<0.05$. long-term memory of emotionally arousing events. This hypothesis is rooted in research suggesting that emotional memories can be stored for the long-term after a single experience or trial (Christianson, 1992), and the BLA interacts with multiple memory systems in the consolidation of long-term memory (McGaugh et al., 2002). Stress hormones, such as adrenaline and glucocorticoids, enhance memory consolidation in rats and humans when administered immediately after training (Gold and van Buskirk, 1975; Liang et al., 1986; Roozendaal et al., 1996; Okuda et al., 2004; Roozendaal et al., 2004; Miranda et al., 2008). Importantly, the effect of these stress hormones depends upon $\beta$-adrenoceptor activity in the BLA (Packard et al., 1995; McGaugh, 2004).

The BLA may influence rACC Arc and CamKII $\alpha$ levels through effects on transport, translation, or degradation of the proteins. CamKII $\alpha$ mRNA, like Arc, is found in stimulated spines of hippocampal neurons (Havik et al., 2003; Moga et al., 2004). In fact, the mRNA for both proteins is packaged in the same granule for transport to the dendrites (Gao et al., 2008). Both have internal ribosomal entry sites (IRES) that might add a translational advantage (Pinkstaff et al., 2001; Svitkin et al., 2005; Dyer et al., 2003). Finally, both Arc and CamKII $\alpha$ have a $3^{\prime}$-UTR intron downstream of their natural stop codon, which has been shown to induce nonsense-mediated decay of Arc, potentially allowing for specific control of breakdown of the mRNAs (Giorgi et al., 2007). In contrast, c-Fos is a somatically localized protein. Consistent with previous findings that c-Fos is increased by novelty or electrical stimulation (Sagar et al., 1988), it was demonstrated here that cFos expression was increased in the rACC following IA training. However, post-training manipulations of the BLA did not further affect c-Fos protein expression as it did Arc and CaMKII $\alpha$. These results suggest that the increases and decreases found in Arc and CaMKII $\alpha$ protein expression in rACC are not merely a result of a general increase in cellular activity due to training. The selective change in levels of locally translated proteins supports the hypothesis that the BLA modulates local translation of synaptic plasticity-associated proteins but, because protein levels were measured in lysates made from the rACC, this result does not exclude the possibility that translation occurs elsewhere in the cell. Additionally, the observed differences may mark preferential degradation of Arc and CamKII $\alpha$.

While these findings suggest that the BLA influences synaptic plasticity in the hippocampus and rACC, they do not exclude the possibility that the interactions that cause the changes in protein expression are bidirectional. Ploski and colleagues (2008) observed increases in Arc mRNA and protein expression in the lateral amygdala (LA) after auditory fear conditioning and by long-term potentiation-inducing stimulation. They further showed that the expression of Arc protein in the LA is important for long-term memory by blocking the expression of Arc protein with antisense ODN infusions into the LA (Ploski et al., 2008). Therefore, while the BLA modulates synaptic plasticity in efferent regions of the brain, transient plasticity may simultaneously 


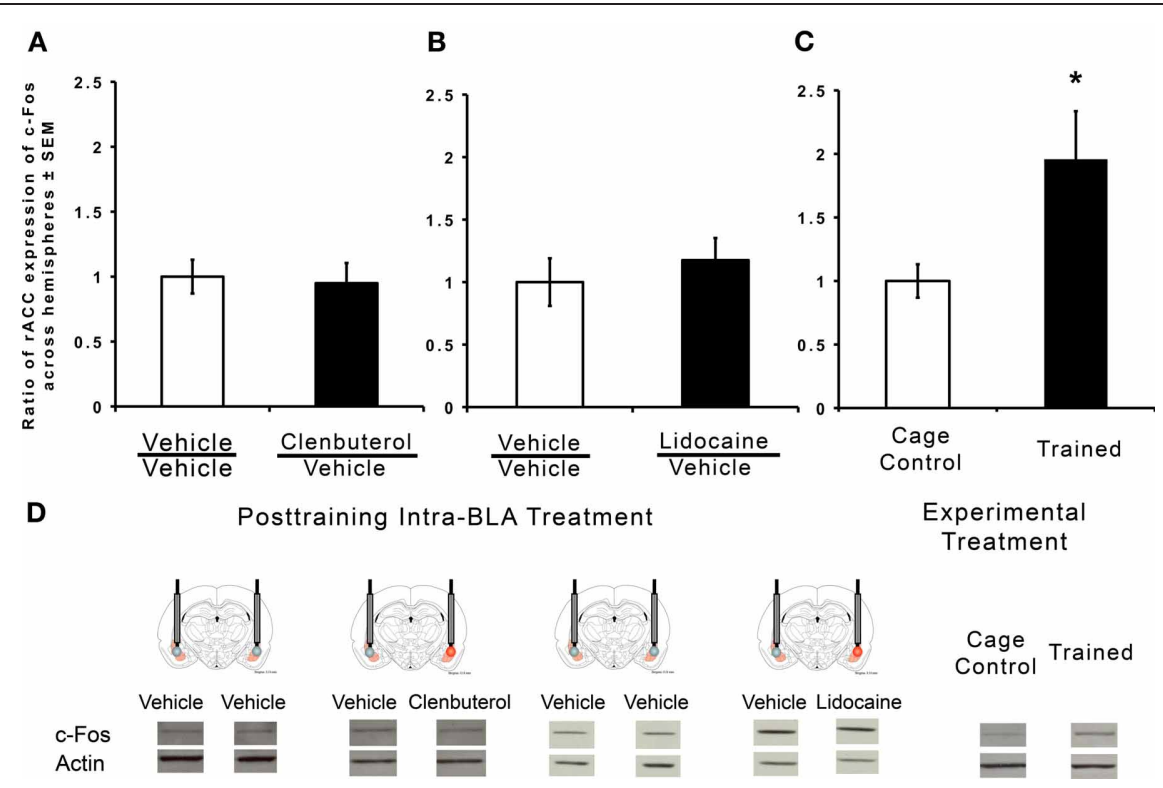

FIGURE 5 | Memory-modulating intra-BLA infusions of clenbuterol and lidocaine did not affect rACC expression of c-Fos. Western immunoblotting was used to quantify protein expression in the rACC following inhibitory avoidance training. c-Fos was normalized to actin by calculating the ratio of band density of c-Fos to that of actin, and then expressed as a percentage of normalized cage control values.

(A) Intra-BLA infusions of clenbuterol immediately following training did not produce a significant increase in c-Fos expression in the rACC
( $m=0.94 \pm 0.15, n=5$ ) when compared to vehicle-treated rats ( $m=1.00 \pm 0.13, n=7 ; p=0.40$ ). (B) Intra-BLA infusions of lidocaine immediately following training did not produce a significant increase in c-Fos expression in the $\mathrm{rACC}(m=1.17 \pm 0.17, n=7)$ when compared to vehicle-treated animals ( $m=1.00 \pm 0.19, n=6 ; p=0.26$ ). (C) c-Fos protein expression was significantly increased in the rACC of rats trained on the inhibitory avoidance task $(m=1.96 \pm 0.38, n=4)$ when compared to untrained rats $(m=1.00 \pm 0.13, n=4)$. ${ }^{*} p<0.05$ occur within the BLA. Other regions of the brain could, likewise, influence that plasticity. For example, lesions of the BLA block the memory enhancement produced by infusions of a glucocorticoid receptor agonist into the $\mathrm{mPFC}$. Phosphorylation of the extracellular-regulated protein kinase (Erk1/2) is increased within the BLA of rats treated with intra-mPFC infusions of the GR agonist and interference of this with intra-BLA infusions of a MEK inhibitor blocked the enhancement of memory produced by intra-mPFC infusions of the GR agonist (Roozendaal et al., 2009). These results suggest that the BLA and $\mathrm{mPFC}$ interact in a bidirectional manner to influence memory consolidation.

The present findings support the hypothesis that the BLA modulates protein expression in other areas of the brain to influence long-term memory. Stimulation of $\beta$-adrenoceptors in the BLA increases, and temporary inactivation of the BLA decreases expression of both Arc and CaMKII $\alpha$. Both proteins are critically involved in memory consolidation and synaptic plasticity. These results indicate that the modulation of Arc protein by the BLA can occur through a process that is conserved across at least two brain regions: the hippocampus and the rACC. The present results also indicate that $\mathrm{CaMKII} \alpha$, another synaptically localized plasticity-related protein, can be modulated by pharmacological stimulation of the BLA. The finding that c-Fos was not significantly affected by the same pharmacological stimulation of the BLA suggests that activation of $\beta$-adrenoceptors in the BLA does not simply lead to a general increase in neuronal activity in the rACC. The specific changes in synaptic proteins may reflect a synapse-specific influence of the BLA on neurons in the rACC. These findings can provide a framework for understanding the mechanisms by which arousal-induced activation of the amygdala modulates consolidation of long-term memory and synaptic plasticity that is distributed across brain regions.

\section{REFERENCES}

Aslam, N., Kubota, Y., Wells, D., and Shouval, H. Z. (2009). Translational switch for long-term maintenance of synaptic plasticity. Mol. Syst. Biol. 5, 284.

Bramham, C. R., Worley, P. F., Moore, M. J., and Guzowski, J. F. (2008). The immediate early gene Arc/Arg3.1: regulation, mechanisms, and function. J. Neurosci. 28, 11760-11767.
Christianson, S-A. (1992). Handbook of Emotion and Memory: Current Research and Theory. New Jersey, NJ: Erlbaum.

Coleman-Mesches, K., and McGaugh, J. L. (1995). Differential effects of pretraining inactivation of the right or left amygdala on retention of inhibitory avoidance training. Behav. Neurosci. 109, 642-647.

Dyer, J. R., Michel, S., Lee, W., Castellucci, V. F., Wayne, N. L., and Sossin, W. S. (2003). An activity-dependent switch to capindependent translation by eIF4E dephosphorylation. Nat. Neurosci. 6, 219-220.

Ferry, B., and McGaugh, J. L. (1999). Clenbuterol administration into the basolateral amygdala posttraining enhances retention in an inhibitory avoidance task. Neurobiol. Learn. Mem. 72, 8-12.
Gallagher, M., Kapp, B. S., Musty, R. E. and Driscoll, P. A. (1977). Memory formation: evidence for a specific neurochemical system in the amygdala. Science 198, 423-425.

Gao, Y., Tatavarty, V., Korza, G., Levin, M. K., and Carson, J. H. (2008). Multiplexed dendritic targeting of $\alpha$ calcium calmodulin-dependent protein kinase II, neurogranin, and activity-regulated cytoskeletonassociated protein RNAs by the 
A2 pathway. Mol. Biol. Cell 19, 2311-2327.

Giorgi, C., Yeo, G. W., Stone, M. E., Katz, D. B., Burge, C., Turrigiano, G., and Moore, M. J. (2007). The EJC factor eIF4AIII modulates synaptic strength and neuronal protein expression. Cell 130, 179-191.

Gold, P. E., and van Buskirk, R. (1975). Facilitation of time-dependent memory processes with posttrial epinephrine injections. Behav. Biol. $13,145-153$.

Guzowski, J. F., Lyford, G. L., Stevenson, G. D., Houston, F. P., McGaugh, J. L., Worley, P. F., and Barnes, C. A. (2000). Inhibition of activity-dependent Arc protein expression in the rat hippocampus impairs the maintenance of long-term potentiation and the consolidation of longterm memory. J. Neurosci. 20, 3993-4001.

Hatfield, T., and McGaugh, J. L. (1999). Norepinephrine administration into the basolateral amygdala posttraining enhances retention in the water-maze task, Neurobiol. Learn. Mem. 71, 232-239.

Havik, B., Rokke, H., Bardsen, K., Davanger, S., and Bramham, C. R. (2003). Bursts of high-frequency stimulation trigger rapid delivery of pre-existing a-CaMKII mRNA to synapses: a mechanism in dendritic protein synthesis during long-term potentiation in adult awake rats, Eur. J. Neurosci. 17, 2679-2689.

Holloway, C. M., and McIntyre, C. K. (2011). Post-training disruption of Arc protein expression in the anterior cingulated cortex impairs long-term memory for inhibitory avoidance training. Neurobiol. Learn. Mem. 95, 425-432.

Kelly, M. P., and Deadwyler, S. A. (2002). Acquisition of a novel behavior induces higher levels of Arc mRNA than does overtrained performance. Neuroscience 110, 617-626.

Kelly, M. P., and Deadwyler, S. A. (2003). Experience-dependent regulation of the immediate-early gene arc differs across brain regions. J. Neurosci. 23, 6443-6451.

Kleim, J. A., Lussnig, E., Schwarz, E. R., Comery, T. A., and Greenough, W. T. (1996). Synaptogenesis and fos expression in the motor cortex of the adult rat after motor skill learning. J. Neurosci. 16, 4529-4535.

Koya, E., Spijker, S., Homberg, J. R., Voorn, P., Schoffelmeer, A.
N., de Vries, T. J., and Smit, A. B. (2005). Molecular reactivity of mesocorticolimbic brain areas of high and low grooming rats after elevated plus maze exposure. Brain Res. Mol. Brain Res. 137, 184-192.

LaLumiere, R. T., Buen, T. V., and McGaugh, J. L. (2003). Posttraining intra-baslolateral amygdala infusions of norepinephrine enhance consolidation of memory for contextual fear conditioning. $J$. Neurosci. 23, 6754-6758.

Liang, K. C., Juler, R. G., and McGaugh, J. L. (1986). Modulating effects of posttraining epinephrine on memory: involvement of the amygdala noradrenergic system. Brain Res. $368,125-133$.

Malin, E. L., Ibrahim, D. Y., Tu, J. W., and McGaugh, J. L. (2007) Involvement of the rostral anterior cingulate cortex in consolidation of inhibitory avoidance memory: interaction with the basolateral amygdala. Neurobiol. Learn. Mem. 87, 295-302.

Malin, E. L., and McGaugh, J. L. (2006). Differential involvement of the hippocampus, anterior cingulate cortex, and the basolateral amygdala in memory for context and footshock. Proc. Natl. Acad. Sci. U.S.A. 103, 1959-1963.

McDonald, A. J. (1991). Organization of amygdaloid projections to the prefrontal cortex and associated striatum in the rat. Neuroscience 44, $1-14$.

McGaugh, J. L. (2004). The amygdala modulates the consolidation of memories of emotionally arousing experiences. Annu. Rev. Neurosci. $27,1-28$.

McGaugh, J. L., McIntyre, C. K., and Power, A. E. (2002). Amygdala modulation of memory consolidation: interaction with other brain systems. Neurobiol. Learn. Mem. 78, 539-552.

McIntyre, C. K., Hatfield, T., and McGaugh, J. L. (2002). Amygdala norepinephrine levels after training predict inhibitory avoidance retention performance in rats. Eur. J. Neurosci. 16, 1223-1226.

McIntyre, C. K., Miyashita, T., Setlow, B., Marjon, K. D., Steward, O., Guzowksi, J. F., and McGaugh, J. L. (2005). Memory-influencing intra-basolateral amygdala drug infusions modulate expression of Arc protein in the hippocampus. Proc. Natl. Acad. Sci. U.S.A. 102, 10718-10723.

McReynolds, J. R., Donowho, K., Abdi, A., McGaugh, J. L., Roozendaal, B., and McIntyre, C. K. (2010).
Memory-enhancing corticosterone treatment increases amygdala norepinephrine and Arc protein expression in hippocampal synaptic fractions. Neurobiol. Learn. Mem. 93 312-321.

Mikkelsen, J. D., and Larsen, M H. (2006). Effects of stress and adrenalectomy on activity-regulated cytoskeleton protein (Arc) gene expression. Neurosci. Lett. 403, 239-243.

Miranda, M. I., LaLumiere, R. T., Buen, T. V., Bermudez-Rattoni, F., and McGaugh, J. L. (2003). Blockade of noradrenergic receptors in the basolateral amygdala impairs taste memory. Eur. J. Neurosci. 18, 2605-2610.

Miranda, M. I., and McGaugh, J. L. (2004). Enhancement of inhibitory avoidance and conditioned taste aversion memory with insular cortex infusions of 8-Br-cAMP: involvement of the basolateral amygdala. Learn. Mem. $11,312-317$.

Miranda, M. I., Quirarte, G. L., Rodriguez-Garcia, G., McGaugh, J. L., and Roozendaal, B. (2008). Glucocorticoids enhance taste aversion memory via actions in the insular cortex and basolateral amygdala. Learn. Mem. 15, 468-476.

Moga, D. E., Calhoun, M. E., Chowdhury, A., Worley, P., Morrison, J. H., and Shapiro, M. L. (2004). Activity-regulated cytoskeletal-associated protein is localized to recently activated excitatory synapses. Neuroscience 125, $7-11$.

Montag-Sallaz, M., and Montag, D. (2003). Learning-induced arg3.1/arc mRNA expression in the mouse brain. Learn. Mem. 10, 99-107.

Okuda, S., Roozendaal, B. and McGaugh, J. L. (2004). Glucocorticoid effects on object recognition memory require training-associate emotional arousal. Proc. Natl. Acad. Sci. U.S.A. 101, 853-858.

Ons, S., Martí, O., and Armario, A. (2004). Stress-induced activation of the immediate early gene Arc (activity-regulated cytoskeletonassociated protein) is restricted to telencephalic areas in the rat brain: relationship to $\mathrm{c}$-fos mRNA. $J$. Neurochem. 89, 1111-1118.

Otmakhov, N., Tao-Cheng, J., Carpenter, S., Asrican, B., Dosemeci, A., Reese, T. S., and Lisman, J. (2004). Persistent accumulation of calcium/calmodulin-dependent protein kinase II in dendritic spines after induction of NMDA receptor-dependent chemical longterm potentiation. J. Neurosci. 24, 9324-9331.

Ouyang, Y., Rosenstein, A., Kreiman, G., Schuman, E. M., and Kennedy, M. B. (1999). Tetanic stimulation leads to increased accumulation of $\mathrm{Ca} 2+/$ calmodulin-dependent protein kinase II via dendritic protein synthesis in hippocampal neurons. J. Neurosci. 19, 7823-7833.

Packard, M. G., Cahill, L., and McGaugh, J. L. (1994). Amygdala modulation of hippocampaldependent and caudate nucleus-dependent memory processes. Proc. Natl. Acad. Sci. U.S.A. 91, 8477-8481.

Packard, M. G., Williams, C. L., Cahill, L., and McGaugh, J. L. (1995). "The anatomy of a memory modulatory system: from periphery to brain," in Neurobehavioral Plasticity: Learning, Development, and Response to Brain Insults, eds N. E. Spear, L. P. Spear, and M. L. Woodruff (New Jersey, NJ: Erlbaum), 149-184.

Paxinos, G., and Watson, C. (2005). The Rat Brain in Stereotaxic Coordinates ( $\left.5^{\text {th }} E d n\right)$. San Diego, CA: Academic Press.

Pinkstaff, J. K., Chappell, S. A., Mauro, V. P., Edelman, G. M., and Krushel, L. A. (2001). Internal initiation of translation of five dendritically localized neuronal mRNAs. Proc. Natl. Acad. Sci. U.S.A. 98, 2770-2775.

Ploski, J. E., Pierre, V. J., Smucny, J., Park, K., Monsey, M. S., Overeen, K. A., and Schafe, G. E. (2008). The activity regulated cytoskeletalassociated protein (Arc/Arg3.1) is required for memory consolidation of pavlovian fear conditioning in the lateral amygdala. J. Neurosci. 28, 12383-12395

Quirarte, G. L., Galvez, R., Roozendaal, B., and McGaugh, J. L. (1998). Norepinephrine release in the amygdala in response to footshock and opioid peptidergic drugs. Brain Res. 808, 134-140.

Quirarte, G. L., Roozendaal, B., and McGaugh, J. L. (1997). Glucocorticoid enhancement of memory storage involves noradrenergic activation in the basolateral amygdala. Proc. Natl. Acad. Sci. U.S.A. 94, 14048-14053.

Roesler, R., Roozendaal, B., and McGaugh, J. L. (2002) Basolateral amygdala lesions block the memory-enhancing effect of 8 - $\mathrm{Br}$ cAMP infused into the entorhinal cortex of rats after training. Eur. J. Neurosci. 15, 905-910.

Roozendaal, B., Castello, N. A., Vedana, G., Barsegyan, A., and McGaugh, J. 
L. (2008). Noradrenergic activation of the basolateral amygdala modulates consolidation of object recognition memory. Neurobiol. Learn. Mem. 90, 576-579.

Roozendaal, B., Hahn, E. L., Nathan, S. V., de Quervain, D. J.-F., and McGaugh, J. L. (2004). Glucocorticoid effects on memory retrieval require concurrent noradrenergic activity in the hippocampus and basolateral amygdala. J. Neurosci. 24, 8161-8169.

Roozendaal, B., McReynolds, J. R., van der Zee, E. A., Lee, S., McGaugh, J. L., and McIntyre, C. K. (2009). Glucocorticoid effects on memory consolidation depend on functional interactions between the medial prefrontal cortex and basolateral amygdala. J. Neurosci. 29, 14299-14308.

Roozendaal, B., Portillo-Marquez, G., and McGaugh, J. L. (1996). Basolateral amygdala lesions block glucocortoid-induced modulation of memory for spatial learning. Behav. Neurosci. 110, 1074-1083.

Sagar, S. M., Sharp, F. R., and Curran, T. (1988). Expression of c-fos protein in brain: metabolic mapping at the cellular level. Science 240, 1328-1333.

Sarter, M., and Markowitsch, H. J. (1983). Convergence of basolateral amygdaloid and mediodorsal thalamic projections in different areas of the frontal cortex in the rat. Brain Res. Bull. 10, 607-622.

Silva, A. J., Paylor, R., Wehner, J. M., and Tonegawa, S. (1992b). Impaired spatial learning in alpha-calciumcalmodulin kinase II mutant mice. Science 257, 206-211.

Silva, A. J., Stevens, C. F., Tonegawa, S., and Wang, Y. (1992a). Deficient hippocampal long-term potentiation in alpha-calcium-calmodulin kinase II mutant mice. Science 257, 201-206.

Sripanidkulchai, K., Sripanidkulchai, B., and Wyss, J. M. (1984). The cortical projection of the basolateral amygdaloid nucleus in the rat: a retrograde flourescent dye study. $J$. Comp. Neurol. 229, 419-431.

Steward, O., and Worley, P. (2002). Local synthesis of proteins at synaptic sites on dendrites: role in synaptic plasticity and memory consolidation? Neurobiol. Learn. Mem. 78, 508-527.

Svitkin, Y. V., Herdy, B., Costa-Mattioli, M., Gingras, A., Raught, B., and Sonenberg, N. (2005). Eukaryotic translation initiation factor $4 \mathrm{E}$ availability controls the switch between cap-dependent and internal ribosomal entry site-mediated translation. Mol. Cell Biol. 25, 10556-10565.

Vazdarjanova, A., and McGaugh, J. L. (1999) Basolateral amygdala is involved in modulating consolidation of memory for classical fear conditioning. J. Neurosci. 19, 6615-6622.

Yin, Y., Edelman, G. M., and Vanderklish, P. W. (2002). The brain-derived neurotrophic factor enhances synthesis of Arc in synaptoneurosomes. Proc. Natl. Acad. Sci. U.S.A. 99, 2368-2373.
Conflict of Interest Statement: The authors declare that the research was conducted in the absence of any commercial or financial relationships that could be construed as a potential conflict of interest.

Received: 17 February 2012; paper pending published: 28 February 2012; accepted: 31 March 2012; published online: 19 April 2012.

Citation: Holloway-Erickson CM, McReynolds JR and McIntyre CK (2012) Memory-enhancing intra-basolateral amygdala infusions of clenbuterol increase Arc and CaMKIIL protein expression in the rostral anterior cingulate cortex. Front. Behav. Neurosci. 6:17. doi: 10.3389/fnbeh.2012.00017 Copyright (C) 2012 Holloway-Erickson, McReynolds and McIntyre. This is an open-access article distributed under the terms of the Creative Commons Attribution Non Commercial License, which permits non-commercial use, distribution, and reproduction in other forums, provided the original authors and source are credited. 